\title{
Maternal and perinatal outcome in patients with preterm labor pains receiving tocolytic therapy
}

\author{
Tanya Rajpal ${ }^{1 *}$, Pooja Patil ${ }^{1}$, Priyanka Sharma ${ }^{2}$, Nishi Mitra ${ }^{1}$
}

\begin{abstract}
${ }^{1}$ Department of Obstetrics and Gynaecology, LN Medical College and JK Hospital, Bhopal, Madhya Pradesh, India ${ }^{2}$ Department of Obstetrics and Gynaecology, Rajasthan Hospital, Jaipur, Rajasthan, India
\end{abstract}

Received: 24 May 2021

Revised: 07 June 2021

Accepted: 08 June 2021

\section{*Correspondence:}

Dr. Tanya Rajpal,

E-mail: trajpal5@gmail.com

Copyright: $@$ the author(s), publisher and licensee Medip Academy. This is an open-access article distributed under the terms of the Creative Commons Attribution Non-Commercial License, which permits unrestricted non-commercial use, distribution, and reproduction in any medium, provided the original work is properly cited.

\begin{abstract}
Background: The objective of this study was to assess the maternal and neonatal outcomes in patients with preterm labor pains and also to correlate threatened abortion with preterm pains.

Methods: The study conducted was a prospective observational study. 100 cases of preterm labor admitted to JK hospital, Bhopal over a period of 2 years with singleton gestation between 28 to $36^{+6}$ weeks were included.

Results: Maximum preterm deliveries were in the late preterm group (80\%). Correlation of preterm labor with threatened abortion was not significant $(\mathrm{OR}=1.03$; $\mathrm{p}>0.05)$. $42 \%$ cases delivered vaginally and $58 \%$ by LSCS. Prolongation of pregnancy after tocolytic therapy was upto 12 hours in 35\% cases, 12-24 hours in $40 \%$ cases and $>24$ hours in only $15 \%$ cases. The most commonly encountered neonatal complication was RDS, $37 \%$, out of which $9 \%$ required resuscitation at birth and $8 \%$ required ventilator support followed by jaundice in $23 \%$, sepsis in $3 \%$ cases and NEC in $4 \%$ cases. The association between gestational age and requirement of resuscitation at birth $\left(X^{2}=19.9 ; p=0.00\right)$, need of ventilator $\left(X^{2}=12.6 ; p=0.002\right)$ and neonatal $\operatorname{RDS}\left(X^{2}=6.9 ; p=0.031\right)$ was found to be significant.

Conclusions: Preterm births are major obstetric problem that have an extensive impact on neonatal morbidity and mortality.
\end{abstract}

Keywords: Preterm birth, Gestational age, Threatened abortion, NICU, RDS

\section{INTRODUCTION}

Preterm births constitute about $85 \%$ of perinatal morbidity and mortality and its incidence is $7-12 \%$ of all deliveries. ${ }^{1}$ Preterm births every year are estimated to be around 15 million and out of these nearly 1 million die due to serious birth complications. ${ }^{2}$ The incidence is rising because of increased numbers of multiple pregnancies due to assisted reproductive techniques (ART), working mothers, increased psychological stress and iatrogenic prematurity. The earlier the onset of labor the more likely are the chances of pathological process being implicated.

The outcome of preterm births depend upon duration of gestation, fetal presentation, duration of latent period, fetal weight, development of chorioamnionitis and most importantly management of patients. The management depends upon likelihood of infection, gestational age and availability of NICU facilities. Preterm labor and births are challenging obstetric complications for the obstetrician, as are the preterm neonates for pediatricians. The risk factors include extremes of maternal age $<20$ years and $>40$ years, low socioeconomic status, previous history of abortion or preterm delivery or associated uterine anomaly. Preterm births have a significant cost implication due to the prolonged hospital stay, NICU and long term complications because of the disabilities. ${ }^{3,4}$ Hence it was important to ascertain the maternal and neonatal outcome of preterm birth and also the resources available in nurseries. 


\section{METHODS}

A prospective observational study was carried out on all patients with singleton pregnancy who were admitted to labor room of JK hospital, Bhopal with gestational age between 28 to $36^{+6}$ weeks with preterm labor pains. The study was carried out from April 2019 to October 2020. A detailed history, complete physical and obstetric examination and routine investigations were done for all women. These women were given pre-hydration, antibiotics, tocolytics and corticosteroids as per standard protocols. Uterine contractions and foetal heart rate and maternal vital signs were monitored as per protocol. An attempt was made to find correlation of threatened abortion with preterm labor pains. Main outcomes that were studied which included prolongation of time to delivery, mode of delivery, maternal complications and neonatal outcome in terms of gestational age, Apgar score, birth weight, NICU admission and need for ventilator, complications. Microsoft excel and R studio (open source analytical tool $\mathrm{V}$ 1.2.335) was used to perform the basic calculation, presentation and statistical analysis of data.

\section{RESULTS}

In our study, majority of women with preterm labor were in the age group $<25$ years, while only 2 were aged $>35$ years. Mean age of women in our study was 25.61 years. Majority of cases of preterm labor were nullipara, 59\% followed by para 1 constituting $28 \%$, para 2 were $9 \%$ and minimum deliveries were in women with parity 3 constituting only $4 \%$. Maximum preterm deliveries were in the late PT $(80 \%)$ followed by moderate preterm $(12 \%)$ and least in the very PT group (8\%).

As shown in Table 1, the correlation of risk of preterm delivery with history of threatened abortion in present pregnancy according to gestational age, the $\mathrm{p}$ value $>0.05$ was non-significant. However an $\mathrm{OR}=1.03$ indicates some positive association between the two.

In our study duration of prolongation of pregnancy after tocolytic therapy was upto 12 hours in 35\% cases, 12-24 hours in $40 \%$ cases and $>24$ hours in only $15 \%$ cases (Figure 1).

Table 2 shows prolongation of labor with tocolytic therapy. Maximum prolongation of labor that was achieved in maximum number of cases was 12-24 hours in $40 \%$ cases, majority in late PT $(36.3 \%)$ group, followed by prolongation upto 12 hours in $35 \%$ cases, majority in late PT group $(37.5 \%)$ and $>24$ hours in $15 \%$ cases, again majority of cases in late PT $(13.8 \%)$ group. However, on associating the duration of prolongation labor with respect to gestational age, no significant difference could be found between them. Hence, duration of prolongation of labor in cases undergoing tocolytic therapy is independent of the gestational age.

Table 1: Correlation of threatened abortion with risk of preterm labor.

\begin{tabular}{|lll|}
\hline Preterm labor (in weeks) & Threatened abortion & No \\
\hline Very PT (28-32) & Yes & 7 \\
\hline Moderate+late PT (32-37) & 1 & 82 \\
\hline
\end{tabular}

$\mathrm{OR}=1.03$; also non-significant association as $\mathrm{p}$ value $>0.05$.

Table 2: Association of prolongation of labor with gestational age.

\begin{tabular}{|c|c|c|c|c|c|}
\hline \multirow{2}{*}{\multicolumn{2}{|c|}{ Prolongation of labor (in hours) }} & \multicolumn{3}{|l|}{ Preterm } & \multirow{2}{*}{ Total } \\
\hline & & \multirow{2}{*}{$\begin{array}{l}\text { Late PT } \\
11\end{array}$} & \multirow{2}{*}{$\begin{array}{l}\text { Moderate PT } \\
3\end{array}$} & \multirow{2}{*}{ Very PT } & \\
\hline$>24(\mathrm{~N}-15)$ & Count & & & & \\
\hline$>24(\mathrm{~N}=15)$ & $\%$ & 13.8 & 25.0 & 12.5 & 15.0 \\
\hline \multirow{2}{*}{$12-24(N=40)$} & Count & 29 & 4 & 7 & 40 \\
\hline & $\%$ & 36.3 & 33.3 & 87.5 & 40.0 \\
\hline \multirow{2}{*}{ Upto $12(\mathrm{~N}=35)$} & Count & 30 & 5 & 0 & 35 \\
\hline & $\%$ & 37.5 & 41.7 & 0.0 & 35.0 \\
\hline \multirow{2}{*}{ None $(\mathbf{N}=10)$} & Count & 10 & 0 & 0 & 10 \\
\hline & $\%$ & 12.5 & 0.0 & 0.0 & 10.0 \\
\hline \multirow{2}{*}{ Total } & Count & 80 & 12 & 8 & 100 \\
\hline & $\%$ & 100.0 & 100.0 & 100.0 & 100.0 \\
\hline \multirow{2}{*}{\multicolumn{2}{|c|}{ Pearson Chi-square }} & Value & Df & $\mathrm{P}$ value & Result \\
\hline & & 11.439 & 6 & 0.076 & Non-significant \\
\hline
\end{tabular}


Table 3: Neonatal complications summary according to gestational age.

\begin{tabular}{|llllllll|}
\hline Gestational age & Resuscitation & NICU & Ventilator & RDS & Jaundice & Sepsis & NEC \\
\hline Late PT (N=80) & 3 & 47 & 3 & 25 & 20 & 1 & 2 \\
\hline Moderate PT (N=12) & 2 & 9 & 2 & 6 & 2 & 1 & 1 \\
\hline Very PT (N=8) & 4 & 7 & 3 & 6 & 1 & 1 & 1 \\
\hline Total & 9 & 63 & 8 & 37 & 23 & 3 & 4 \\
\hline Value & 19.974 & 3.421 & 12.647 & 6.961 & 0.950 & 4.496 & 2.561 \\
\hline Df & 2 & 2 & 2 & 2 & 2 & 2 & 2 \\
\hline P value & 0.000 & 0.181 & 0.002 & 0.031 & 0.622 & 0.106 & 0.278 \\
\hline
\end{tabular}

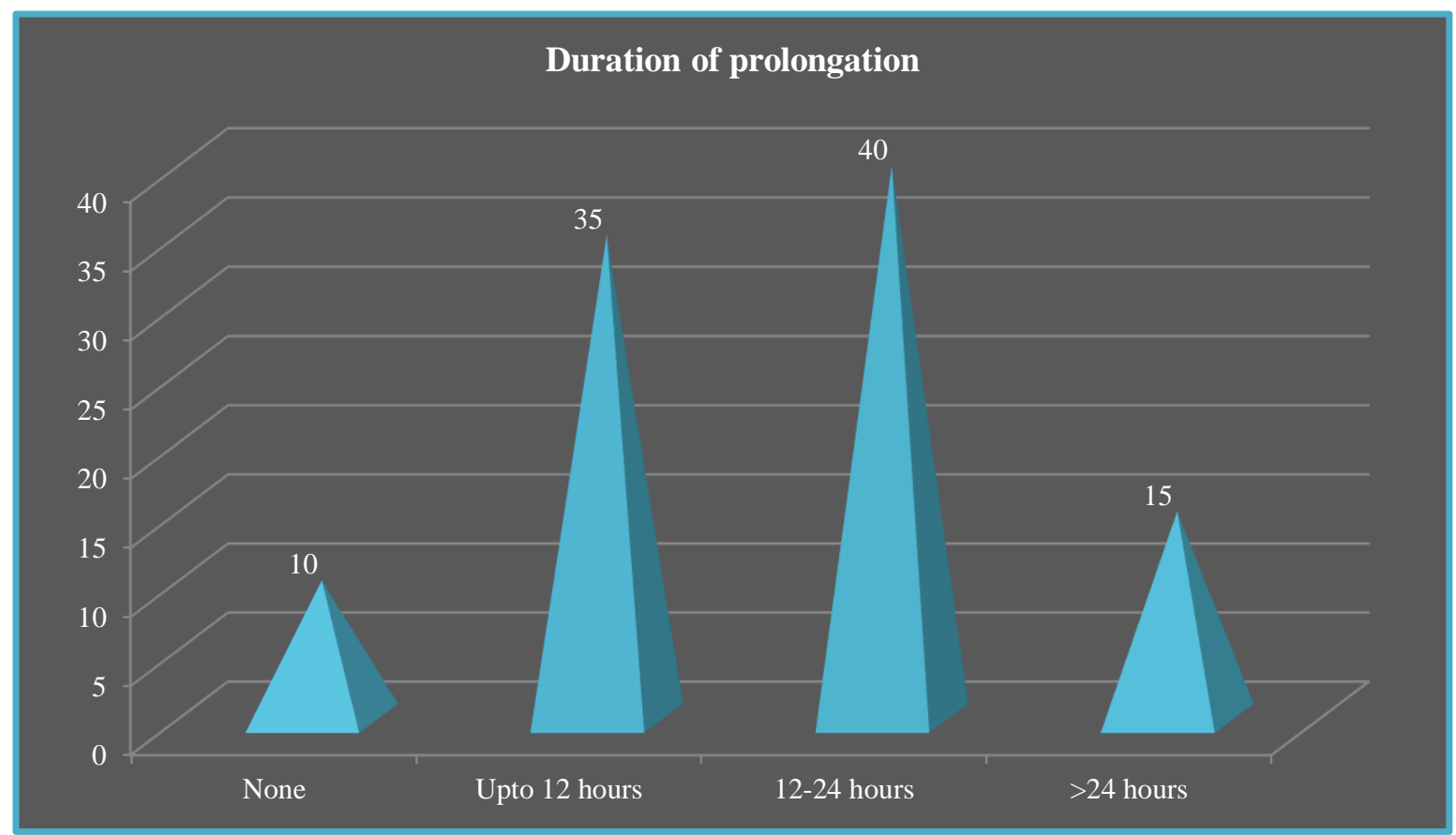

Figure 1: Duration of prolongation of pregnancy after tocolytic therapy.

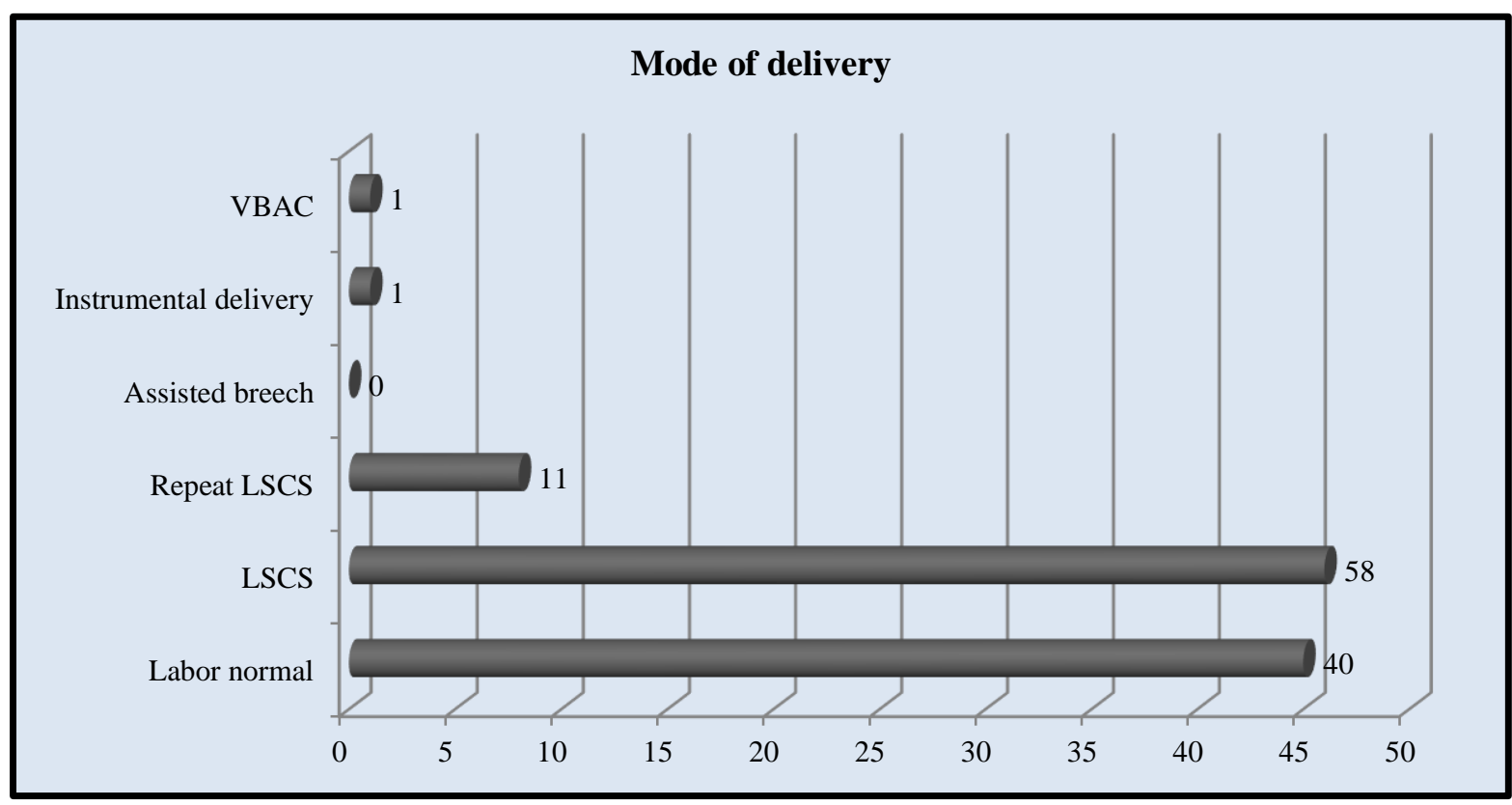

Figure 2: Distribution according to mode of delivery. 


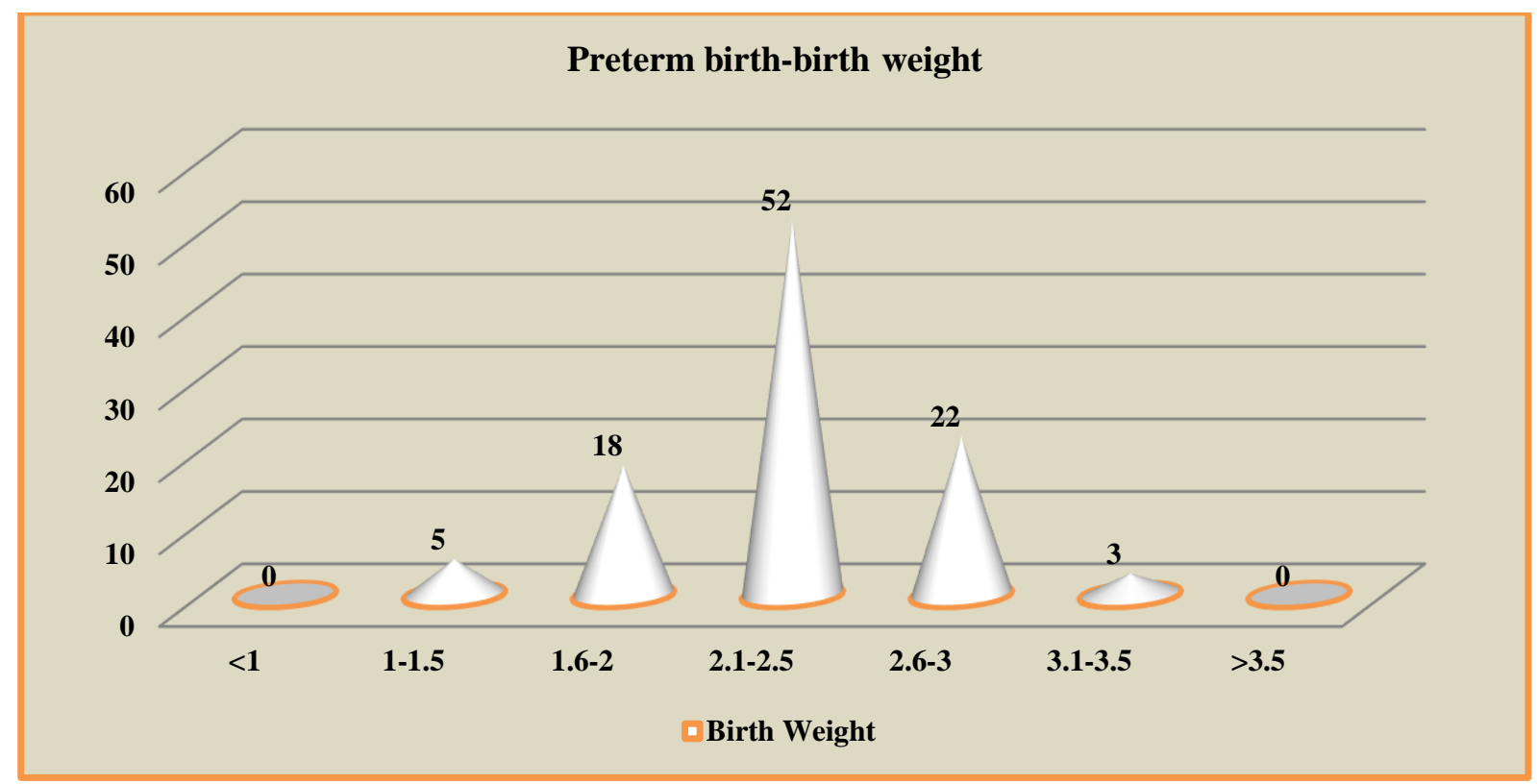

Figure 3: Distribution of cases according to birth weight.

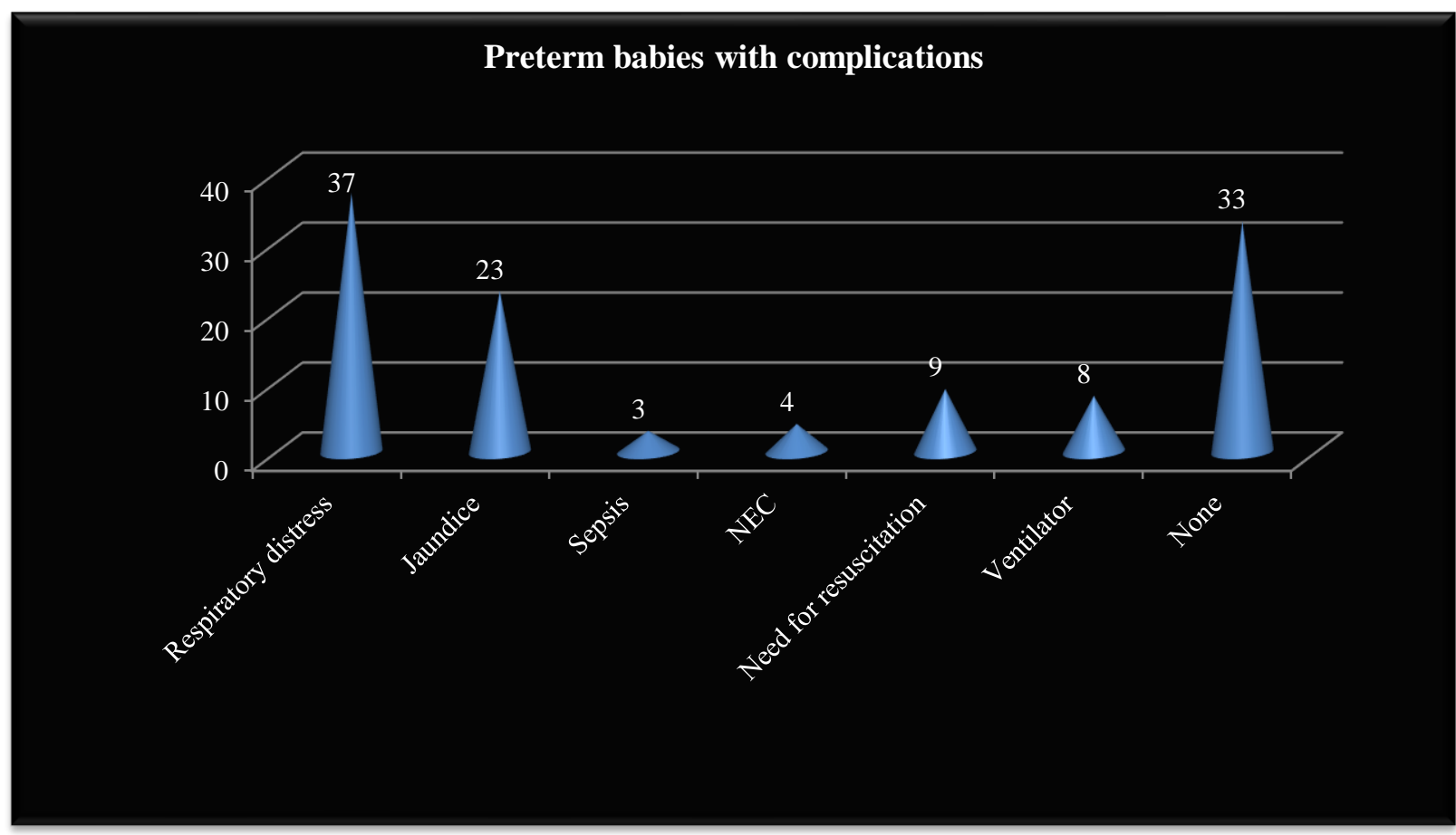

Figure 4: Incidence of neonatal complications in preterm.

Out of the total deliveries, $40 \%$ were vaginal, $1 \%$ instrumental, $1 \%$ VBAC, while out of the $58 \%$ LSCS. $12 \%$ were repeat LSCS (Figure 2).

Figure 3 shows the birth weight distribution of the newborn, according to which maximum cases were found to be in the birth weight range of $2.1-2.5 \mathrm{kgs}$ followed by those in the range $2.6-3 \mathrm{kgs}$. There were no newborn with weight $<1 \mathrm{~kg}$ or $>3.5 \mathrm{kgs}$ in our study.

As shown in Figure 4, the most commonly encountered neonatal complication was RDS, $37 \%$ followed by jaundice in $23 \%$, sepsis in $3 \%$ cases and NEC in $4 \%$ cases. $8 \%$ neonates required ventilator. 
As depicted in Table 3, association between gestational age and requirement of resuscitation at birth ( $\mathrm{p}$ value $<0.05$ ), ventilator ( $\mathrm{p}$ value $<0.05$ ) and neonatal RDS ( $\mathrm{p}$ value $<0.05$ ) was found to be significant. However, neonatal complications such as jaundice, sepsis, NEC were not significantly associated with gestational age ( $\mathrm{p}$ value $>0.05)$.

\section{DISCUSSION}

As discussed above, preterm births are truly a global problem. In spite of the improvements in referral and health facilities the incidence of preterm births is showing a rising trend which can be attributed to the ART procedures, maternal factors like physical and psychological stress.

In a study by Saraswat et al in 2010, odds of having preterm delivery in patients with history of threatened abortion was $\mathrm{OR}=2.05,95 \% \mathrm{CI} 1.76,2.4 .{ }^{5} \mathrm{In}$ another study by Ahmed et al in 2012,15.7\% women out of 89 cases had a history of threatened abortion ( $p$ value $<0.05$ ) which was significant. ${ }^{6}$ In our study out of the 100 cases of preterm birth, $11 \%$ had history of threatened abortion.

In a study by Shehla et al $23.7 \%$ cases delivered by caesarean section, $75.4 \%$ cases delivered vaginally and $00.9 \%$ by assisted vaginal delivery. ${ }^{7}$ In our study 42 deliveries were through vaginal route, out of which 1 was instrumental delivery and 1 was VBAC. Out of the total 58 cesarean sections in our study, 12 were repeat sections in view of previous LSCS.

In a study by Kuzniewicz on outcomes of preterm labor in 2017, depending on the gestational age, the time to deliver differed. In $96 \%$ cases of late preterm labor, that is, >34 weeks, delivery occurred within 48 hours duration. bewteen $31-33$ weeks, delivery occurred in $67 \%$ cases before 48 hours and those admitted at $<31$ weeks, $51.9 \%$ delivered within 48 hours and $77.6 \%$ delivered within 7 days of admission to the hospital. ${ }^{8}$ In a study by Sudhir et al (2016) in $17.75 \%$ cases pregnancy was prolonged upto 2 days, in $14.95 \%$ cases upto 1 week, in $3.73 \%$ cases upto 2 weeks. The prolongation in $5.6 \%$ cases was achieved upto term and in $57.94 \%$ cases which comprised majority if patients, delivered on the day of admission. ${ }^{9}$ In our study, prolongation of labor after tocolytic therapy was upto 12 hours in $35 \%$ cases, $12-24$ hours in $40 \%$ cases and $>24$ hours in only $15 \%$ cases. No prolongation could be achieved in $10 \%$ cases.

Charitha (2010) in her study on the outcome of preterm labor found that $34 \%$ patients were between $34-36$ weeks of gestation, $32 \%$ in $32-34$ weeks, $22 \%$ in $30-32$ weeks and $12 \%$ in $28-30$ weeks gestation. ${ }^{10}$ In our study, late PT (3437 weeks) cases were $80 \%$, those born between $32-34$ weeks constituted $12 \%$ while only $8 \%$ constituted very PT cases (28-32 weeks). None of the cases in our study were in the extremely preterm group. The incidence of perinatal morbidity due to preterm births was $34 \%$. The incidence of RDS in their study was high, in 18 cases, 3 had birth asphyxia, 2 had septicemia. RDS comprised $76 \%$ of total neonatal morbidity. Out of the 18 babies with RDS, 13 received corticosteroids. Out of the two cases of septicemia, one died.

Study by Akhter et al neonatal morbidity was found higher in preterm infants when compared to controls. ICU admission rates in preterm births was noted to be statistically significant. In their study 9 cases had sepsis, 31 RDS and 15 RDS with sepsis. ${ }^{11}$ Refuerzo et al studied the rate of sepsis in preterm cases and found that sepsis was found twice as frequently in cases of moderate PT when compared to late PT, rate of sepsis being $5 \%$ in the former and $2.2 \%$ in the latter. ${ }^{12}$ In our study, 37 babies had RDS, 9 required resuscitation at birth and 8 were on ventilator support. ${ }^{23}$ Infants reported neonatal hyperbilirubinemia clustering on 3rd to 4th day of birth. ${ }^{3}$ Neonates had septicemia and 4 had NEC.

There were limitations in our study. Standard tocolytic therapy was given to patients and hence there was no comparison between efficacy of various tocolytic options for preterm labor. We only evaluated perinatal and shortterm neonatal outcomes, before neonate's and woman's discharge. New hospitalization and complications were not included in our study.

\section{CONCLUSION}

Preterm labor is a major obstetric problem with adverse neonatal outcomes. Positive association is found between history of threatened abortion in present pregnancy with preterm labor pains. A prolongation of 12-24 hours was achieved in maximum number of cases (40 cases) with preterm labor pains. RDS is a major cause of neonatal morbidity. Number of very preterm births has decreased over the years which could be attributed to timely diagnosis and adequate management.

Funding: No funding sources

Conflict of interest: None declared

Ethical approval: The study was approved by the Institutional Ethics Committee

\section{REFERENCES}

1. Norwitz ER, Robinson JN. A systematic approach to the management of preterm labor. Semin Perinatol. 2001;25(4):223-35.

2. Chawanpaiboon S, Vogel JP, Moller AB, Lumbiganon P, Petzold M, Hogan D, et al. Global, regional, and national estimates of levels of preterm birth in 2014: a systematic review and modelling analysis. Lancet Glob Health. 2019;7(1):37-46.

3. Zainal H, Dahlui M, Soelar SA, Su TT. Cost of preterm birth during initial hospitalization: a care provider's perspective. PloS One. 2019;14(6):0211997. 
4. Karnati S, Kollikonda S, Abu-Shaweesh J. Late preterm infants-changing trends and continuing challenges. Int J Pediatr Adolesc Med. 2020;7(1):3644.

5. Saraswat L, Bhattacharya S, Maheshwari A, Bhattacharya S. Maternal and perinatal outcome in women with threatened miscarriage in the first trimester: a systematic review. BJOG Int J Obstetr Gynaecol. 2010;117(3):245-57.

6. Ahmed SR, El-Sammani ME, Al-Sheeha MA, Aitallah AS, Khan FJ, Ahmed SR. Pregnancy outcome in women with threatened miscarriage: a year study. Mater Sociomed. 2012;24(1):26-8.

7. Jamal S, Srivastava R. A retrospective analytical study of the epidemiology and causes of preterm birth. Int J Reprod Contracept Obstet Gynecol. 2017;6(12):5453-7.

8. Kuzniewicz MW, Black L, Walsh EM, Li SX, Greenberg M. Outcomes of admissions for preterm labor. AJP Rep. 2017;7(2):106-13.
9. Sudhir SP, Mishra S. A prospective study of etiology and outcome of preterm labor in a rural medical college. Obg Rev J Obstet Gynecol. 2016;2(4):60-4.

10. Charitha U. To study the etiology and outcome of preterm labor. RGUHS. 2010.

11. Akhter G, Rizvi SM, Hussain SI, Ali F, Ali A. Evaluation of perinatal outcome in preterm labor. Int J Current Res Rev. 2015;7(21):66.

12. Refuerzo JS, Momirova V, Peaceman AM, Sciscione A, Rouse DJ, Caritis SN, et al. Neonatal outcomes in twin pregnancies delivered moderately preterm, late preterm and term. Am J Perinatol. 2010;27(7):537.

Cite this article as: Rajpal $\mathrm{T}$, Patil $\mathrm{P}$, Sharma $\mathrm{P}$, Mitra N. Maternal and perinatal outcome in patients with preterm labor pains receiving tocolytic therapy. Int J Reprod Contracept Obstet Gynecol 2021;10:2632-7. 